\title{
On Gauss-Bonnet Curvatures ${ }^{\star}$
}

Mohammed Larbi LABBI

Mathematics Department, College of Science, University of Bahrain, 32038 Bahrain

E-mail: labbi@sci.uob.bh

Received August 27, 2007, in final form November 15, 2007; Published online December 11, 2007

Original article is available at http://www.emis.de/journals/SIGMA/2007/118/

\begin{abstract}
The (2k)-th Gauss-Bonnet curvature is a generalization to higher dimensions of the $(2 k)$-dimensional Gauss-Bonnet integrand, it coincides with the usual scalar curvature for $k=1$. The Gauss-Bonnet curvatures are used in theoretical physics to describe gravity in higher dimensional space times where they are known as the Lagrangian of Lovelock gravity, Gauss-Bonnet Gravity and Lanczos gravity. In this paper we present various aspects of these curvature invariants and review their variational properties. In particular, we discuss natural generalizations of the Yamabe problem, Einstein metrics and minimal submanifolds.
\end{abstract}

Key words: Gauss-Bonnet curvatures; Gauss-Bonnet gravity; lovelock gravity; generalized Einstein metrics; generalized minimal submanifolds; generalized Yamabe problem

2000 Mathematics Subject Classification: 53C20; 53C25

\section{An introduction to Gauss-Bonnet curvatures}

We shall present in this section several approaches to the Gauss-Bonnet curvatures. For precise definitions and examples the reader is encouraged to consult $[17,18,20]$.

\subsection{Gauss-Bonnet curvatures vs. curvature invariants of Weyl's tube formula}

In a celebrated paper [38] published in 1939, Hermann Weyl proved that the volume of a tube of radius $r$ around an embedded compact $p$-submanifold $M$ of the $n$-dimensional Euclidean space is a polynomial in the radius of the tube as follows:

$$
\operatorname{Vol}(\operatorname{tube}(r))=\sum_{i=0}^{[p / 2]} C(n, p, i) H_{2 i} r^{2 i},
$$

where $C(n, p, i)$ are constants which only depend on the dimension and the codimension of the submanifold $M$, and $H_{2 i}$ are integrals of intrinsic scalar curvatures of the submanifold (GaussBonnet curvatures). A good and complete reference about Weyl's tube formula and related topics is the book of A. Gray [13].

It turns out that $H_{0}$ is the volume of the submanifold, $H_{2}$ is the integral of the usual scalar curvature of the submanifold, the integrand in $H_{4}$ is quadratic in the Riemann tensor and was introduced by Lanczos in 1932 as a possible substitute to Hilbert's Lagrangian in general relativity. The top $H_{p}$ is up to a constant the Euler-Poincaré characteristic of the submanifold if $p$ is even.

All the (total Gauss-Bonnet) curvatures $H_{2 i}$ have important applications in theoretical physics, particularly in (brane world) cosmology. They are by nowadays the subject of intensive studies, where they are known as the Lagrangian of Lovelock gravities or Gauss-Bonnet gravities, see $[8,10,26]$ and the references therein.

\footnotetext{
*This paper is a contribution to the Proceedings of the 2007 Midwest Geometry Conference in honor of Thomas P. Branson. The full collection is available at http://www.emis.de/journals/SIGMA/MGC2007.html
} 


\subsection{Gauss-Bonnet curvatures vs. Gauss-Bonnet integrands}

\subsubsection{From Gaussian curvature to the scalar curvature}

Recall that for a compact 2-dimensional Riemannian manifold $(M, g)$ (a surface) the classical Gauss-Bonnet formula states that the Euler-Poincaré characteristic of $M$ (which is a topological invariant) is determined by the geometry of $(M, g)$ as an integral of the Gaussian curvature of the metric: the 2-dimensional Gauss-Bonnet integrand. It is a scalar function defined on the surface and can be naturally generalized to higher dimensional Riemannian manifolds in the following way:

Let $(M, g)$ be a Riemannian manifold of dimension $n \geq 2$. For $m \in M$ and for a tangent 2-plane $P$ to $M$ at $m$ we define $K(P)$, the sectional curvature at $P$, to be the Gaussian curvature at $m$ of the surface $\exp _{m}(V)$, where $\exp _{m}$ is the exponential map and $V$ is a small neighborhood of 0 in $V$. Recall that the so-obtained surface is totally geodesic at $m$. In this way, we obtain a function $K$ defined on the 2-Grassmannian bundle over $M$. The function $K$ determines a unique symmetric $(2,2)$-double form $R$ that satisfies the first Bianchi identity and having $K$ as its sectional curvature, that is nothing but the standard Riemann curvature tensor. Recall that a symmetric $(2,2)$-double form is a $(0,4)$ tensor which is skew symmetric in the first two arguments and in the last two, and that it is symmetric with respect to the interchange of the first two variables with the last two.

Then one can define the scalar curvature of $M$ by taking the Ricci contraction of $R$ twice. In this sense one can say that the usual scalar curvature is a natural generalization of the two dimensional Gauss-Bonnet integrand to higher dimensions.

\subsubsection{From higher Gauss-Bonnet integrands to Gauss-Bonnet curvatures}

For a compact $(2 p)$-dimensional Riemannian manifold $(M, g)$ the generalized Gauss-Bonnet theorem states that the Euler-Poincaré characteristic of $M$ (which is a topological invariant) is determined by the geometry of $(M, g)$ as an integral of a certain curvature of the metric:

$$
\chi(M)=c(p) \int_{M} h_{2 p} \mathrm{dvol}
$$

where $c(p)$ is a constant and $h_{2 p}$ is a scalar function on the manifold defined using the Riemann curvature tensor of $(M, g)$ : the $(2 p)$-th Gauss-Bonnet integrand, called also the Lipschitz-Killing curvature [11].

Using the same idea as above, we generalize the $(2 p)$-th Gauss-Bonnet integrand (or the $(2 p)$-th Killing-Lipschitz curvature) to dimensions higher than $(2 p)$ as follows.

Let $(M, g)$ be a Riemannian manifold of dimension $n \geq 2 p$. For $m \in M$ and for a tangent (2p)-plane $P$ to $M$ at $m$ we define $K_{2 p}$, called Thorpe's $(2 p)$-th sectional curvature at $P$ [35], to be the Gauss-Bonnet integrand at $m$ of the $(2 p)$-dimensional submanifold $\exp _{m}(V)$, where $\exp _{m}$ and $V$ are as above. Thorpe's tensor $R_{2 p}$ of order $(2 p)$ is then defined to be the unique symmetric $(p, p)$-double form that satisfies the first Bianchi identity and with sectional curvature $K_{2 p}$.

Then one can get scalar curvatures (Gauss-Bonnet curvatures) after taking $(2 p)$-times the Ricci contraction of $R_{2 p}$.

The tensors $R_{2 p}$ are determined by the Riemann curvature tensor $R$ in the following way [36]: For $u_{i}, v_{j}$ tangent vectors at $m \in M$, we have

$$
\begin{aligned}
\frac{(2 p) !}{2^{p}} & R_{2 p}\left(u_{1}, \ldots, u_{2 p}, v_{1}, \ldots, v_{2 p}\right) \\
& =\sum_{\alpha, \beta \in S_{2 p}} \epsilon(\alpha) \epsilon(\beta) R\left(u_{\alpha(1)}, u_{\alpha(2)}, v_{\beta(1)}, v_{\beta(2)}\right) \cdots R\left(u_{\alpha(2 p-1)}, u_{\alpha(2 p)}, v_{\beta(2 p-1)}, v_{\beta(2 p)}\right) .
\end{aligned}
$$


This complicated expression can be considerably simplified using the exterior product of double forms, see the following subsection.

\subsection{Double forms}

A $(p, q)$-double form $\omega\left(x_{1}, x_{2}, \ldots, x_{p}, y_{1}, y_{2}, \ldots, y_{q}\right)$ on $M$ is at each point of the manifold a multilinear form that is skew symmetric with respect to the interchange of any two among the first $p$-arguments (tangent vectors) or the last $q$. If $p=q$ and $\omega$ is invariant with the respect to the interchange of the first $p$-variables with the last $p$, we say that $\omega$ is a symmetric $(p, p)$-double form.

For example, the covariant Riemann curvature tensor is a symmetric $(2,2)$-double form, and Thorpe's tensor $R_{2 p}$ is a symmetric $(2 p, 2 p)$-double form.

Double forms were introduced first by De Rham and then developed in the seventies of the last century by Thorpe [36], Kulkarni [16], Nomizu [29], Gray [12], Kowalski [15], Nasu [28] etc.

Double forms and the more general multi-forms are recently studied in theoretical physics, see [33] and the references therein.

\subsubsection{Algebraic operations on double forms}

A $(p, q)$-double form can be seen alternatively as a section of the tensor product of the bundle of $p$-forms with the one of $q$-forms.

1. The exterior product of double forms is the natural generalization to double forms of the usual exterior product of differential forms:

$$
\left(\theta_{1} \otimes \theta_{2}\right) \cdot\left(\theta_{3} \otimes \theta_{4}\right)=\left(\theta_{1} \wedge \theta_{3}\right) \otimes\left(\theta_{2} \wedge \theta_{4}\right),
$$

where we denoted the exterior product of double forms following Kulkarni [16] by a dot. In the following the dot shall be omitted whenever possible. The well known KulkarniNomizu product of symmetric bilinear forms is a special case of the exterior product, see [6, p. 47].

2. The generalized Hodge star operator [17] is the natural extension to double forms of the usual Hodge star operator on differential forms:

$$
*\left(\theta_{1} \otimes \theta_{2}\right)=\left(* \theta_{1}\right) \otimes\left(* \theta_{2}\right) .
$$

Senovilla [33] considered other interesting extensions of the Hodge star operator to doubleforms and multi-forms by keeping it acting on different factors of the tensor product.

3. The inner product of double forms [17] is defined by declaring

$$
\left\langle\theta_{1} \otimes \theta_{2}, \theta_{3} \otimes \theta_{4}\right\rangle=\left\langle\theta_{1}, \theta_{3}\right\rangle\left\langle\theta_{2}, \theta_{4}\right\rangle .
$$

The exterior product of double forms has the advantage that it makes easier many complicated expressions of Riemannian geometry, as illustrated by the examples below. In the following let $(M, g)$ denote a Riemannian manifold of dimension $n$ and $R$ its Riemann curvature tensor seen as a $(2,2)$-double form.

- Thorpe tensors are just given by $[16,28]$

$$
R_{2 p}=\frac{2^{p}}{(2 p) !} R^{p}
$$

where of course $R^{p}$ is the exterior product of the Riemann curvature tensor $R$ seen as a $(2,2)$-double form. In particular, $R^{n / 2}$ determines the Gauss-Bonnet integrand if the dimension $n$ of the manifold is even. 
- The $(2 k)$-th Gauss-Bonnet curvature of $(M, g)$ can be alternatively defined by [17]:

$$
h_{2 p}=* \frac{1}{(n-2 p) !}\left(g^{n-2 p} R^{p}\right) .
$$

- The curvature operator of the classical Weitzenböck formula acting on $p$-forms is determined by the following double form $[7,21]$.

$$
\left\{\frac{g \mathrm{Ric}}{(p-1)}-2 R\right\} \frac{g^{p-2}}{(p-2) !}
$$

where Ric denotes the Ricci curvature of $(M, g)$.

- The exterior product $g^{k}=g \cdots g$ determines the canonical inner product of differential $k$-forms over $M$.

- Gauss equation for a hypersurface of the Euclidean space which relates the curvature tensor $R$ of the hypersurface to its second fundamental form $B$ can be just written as [13]

$$
R=\frac{1}{2} B^{2}
$$

\subsection{Gauss-Bonnet curvatures vs. symmetric functions in the eigenvalues of the shape operator of a hypersurface of the Euclidean space}

Let $g$ and $B$ denote respectively the first and second fundamental forms of a hypersurface of the Euclidean space. The symmetric functions in the eigenvalues of the operator corresponding to $B$ can be nicely re-formulated using the exterior product and the generalized Hodge star operator as follows [23]

$$
s_{k}=\frac{1}{k !(n-k) !} *\left(g^{n-k} B^{k}\right) .
$$

In particular, if $k=2 p$ is even, Gauss equation shows that $R=\frac{1}{2} B^{2}$. Therefore, all the even powers of $B$ are then intrinsic and consequently $s_{2 p}$ is also intrinsic and coincides up to a constant with the Gauss-Bonnet curvature of the hypersurface as follows:

$$
s_{2 p}=\frac{2^{p}}{(2 p) !(n-2 p) !} *\left(g^{n-2 p} R^{p}\right)=\frac{2^{k}}{(2 k) !} h_{2 k} .
$$

Note that if $k=2 p+1$ is odd then $s_{2 p+1}$ is no longer intrinsic:

$$
s_{2 p+1}=* \frac{g^{n-2 p-1} B^{2 p+1}}{(n-2 p-1) !(2 p+1) !}=* \frac{2^{p} g^{n-2 p-1} R^{p} B}{(n-2 p-1) !(2 p+1) !} .
$$

The previous formula allows one to define the Gauss-Bonnet curvatures of odd order for an arbitrary submanifold as follows:

Definition 1 ([23]). Let $(M, g)$ be an arbitrary $n$-submanifold of a Riemannian manifold $(\tilde{M}, \tilde{g})$ and $N$ a normal vector to $M$. For $1 \leq 2 p+1 \leq n$, we define the $(2 p+1)$-th Gauss-Bonnet curvature of $(M, g)$ at $N$ by

$$
h_{2 p+1}(N)=*\left(\frac{g^{n-2 p-1}}{(n-2 p-1) !} R^{p} B_{N}\right),
$$

where $B$ denotes the vector valued second fundamental form of $M, B_{N}(u, v)=\tilde{g}(B(u, v), N)$ and $R$ is the Riemann curvature tensor of $(M, g)$. 
The $(2 p+1)$-th Gauss-Bonnet curvature is a generalization of the usual mean curvature as for $p=0$ we recover the trace of $B$ :

$$
h_{1}(N)=*\left(\frac{g^{n-1}}{(n-1) !} B_{N}\right)=c B_{N} .
$$

Furthermore, for a submanifold of the Euclidean space, $h_{2 p+1}$ coincides with the higher $(2 p+1)$ th mean curvature defined by Reilly [32].

\subsection{Other aspects of Gauss-Bonnet curvatures and terminology}

The Gauss-Bonnet curvatures, or more precisely closely related invariants, appear naturally in the mathematics and physics literature under different names. For instance, in the context of convex sets they (more precisely their integrals) are known as Minkowski's Quermassintegrale, intrinsic volumes or Steiner functionals. They are called Lipschitz-Killing curvatures (measures) in the case of sets of positive reach, piecewise linear spaces and subanalytic sets. A good compterendu of these different aspects is the review paper of Bernig [5]. These invariants appear also in the study of the "expected Euler characteristic" in the probability literature [1] and in extensions of the Gauss-Bonnet theorem [2].

In theoretical physics Gauss-Bonnet curvatures are known as a Lagrangian of pure Lovelock gravity, Gauss-Bonnet gravity or Lanczos gravity. Precisely, in dimensions higher than four the Lagrangian of Lovelock gravity is [8]

$$
L=\sum_{k=0}^{m} c_{2 k} h_{2 k},
$$

where $h_{0}=1, c_{0}$ is the cosmological constant, the higher $c_{k}$ are arbitrary constants and $h_{2 k}$ (resp. $m$ ) is the $(2 k)$-th Gauss-Bonnet curvature (resp. the integer part of one half the dimension of) the (pseudo) Riemannian manifold under consideration.

Physicists consider the Lovelock gravity as a fascinating extension of general relativity for dimensions higher than four, see for instance [8] and the references therein. The Lagrangian of the so-called pure Lovelock gravity is the Gauss-Bonnet curvature (that is just one term in the summation in (3)). The Lagrangian of the Gauss-Bonnet gravity (called also Lanczos gravity) is extensively studied in theoretical physics, it is obtained by taking only the first three terms in the summation (3), see $[26,8]$ and the references therein.

Finally we make the following comments about terminology. Some authors name all the previous invariants as Killing-Lipschitz curvatures in all categories. In the smooth Riemannian case Killing-Lipschitz curvatures refer originally to the Gauss-Bonnet integrands [11]. On the other hand, there is no standard terminology for the scalar curvatures (that are the GaussBonnet curvatures under study here) generalizing the previous integrands. For instance, in [5, $9,24]$ they are still called Lipschitz-Killing curvatures, in $[3,14]$ they are H. Weyl's invariants, in $[13,32]$ they are called higher mean curvatures, physicists call them dimensionally continued (or extended) Euler densities or Gauss-Bonnet integrands. It was a subtle suggestion made to us by an anonymous referee of the Pacific J. Math. to rather call these higher order scalar curvatures in the smooth (Riemannian) case as the Gauss-Bonnet or Gauss-Bonnet-Weyl curvatures.

\section{Einstein-Lovelock tensors}

The usual Ricci curvature tensor $c R$ is the first Ricci-contraction of the Riemann curvature tensor $R$. The Einstein tensor is the simplest linear combination of the the Ricci tensor and the metric tensor to be divergence free, that is $\frac{1}{2} c^{2} R g-c R$. It is the gradient of the total scalar 
curvature seen as a functional on the space of all Riemannian metrics on the manifold under consideration.

In a similar way, we define a generalized Ricci curvature tensor $c^{2 p-1} R^{p}$ of order $(2 p)$ to be the $(2 p-1)$-th Ricci contraction of Thorpe's tensor $R^{p}$. The Einstein-Lovelock tensor $T_{2 p}$ is a linear combination of the $(2 p)$-th Ricci tensor $c^{2 p-1} R^{p}$ and the metric tensor that is divergence free. Precisely, we define the Einstein-Lovelock tensor $T_{2 p}$ of order $2 p$ by

$$
T_{2 p}=h_{2 p} g-\frac{1}{(2 p-1) !} c^{2 p-1} R^{p} .
$$

For $p=0$, we set $T_{0}=g$. For $p=1, T_{2}$ coincides with the usual Einstein tensor. Furthermore, the tensor $T_{2 k}$ is the gradient of the total $(2 k)$-th Gauss-Bonnet curvature seen as a functional on the space of all Riemannian metrics on a given compact manifold, see the next section.

David Lovelock [25] proved that any divergence-free symmetric $(0,2)$ tensor built from the metric and its first two covariant derivatives are linear combinations of the tensors $T_{2 k}$.

\section{A variational property of the Gauss-Bonnet curvatures}

On a compact manifold, we have the classical total scalar curvature functional:

$$
S(g)=\int_{M} \operatorname{scal}(g) \mu_{g}
$$

The gradient of this Riemannian functional is the Einstein tensor: $\frac{1}{2}$ scal $g$ - Ric. The critical metrics of $S$ once restricted to metrics with unit volume, are the Einstein metrics.

Similar properties hold for the total Gauss-Bonnet curvature functional:

$$
H_{2 k}(g)=\int_{M} h_{2 k}(g) \mu_{g}
$$

as shown by the following theorem:

Theorem 1. Let $(M, g)$ be a compact Riemannian manifold of dimension $n$. For each $k$, such that $2 \leq 2 k \leq n$, the functional $H_{2 k}$ is differentiable, and at $g$ we have

$$
H_{2 k}^{\prime} h=\frac{1}{2}\left\langle h_{2 k} g-\frac{1}{(2 k-1) !} c^{2 k-1} R^{k}, h\right\rangle .
$$

In particular, the gradient of $2 H_{2 k}$ is $T_{2 k}=h_{2 k} g-\frac{1}{(2 k-1) !} c^{2 k-1} R^{k}$.

The previous theorem were first proved by Lovelock [25] using classical tensor analysis, Berger [3] in the case $k=2$, Patterson [30] as a special case of a more general variational formula due to Muto [27] and Bernig [4] proved a more general variational formula for the Lipschitz-Killing curvatures of subanalytic sets. The proof of [19] sketched below is coordinate free and it uses the the formalism of double forms.

Proof. We sketch the proof of the theorem, for more details see [19]. First, the directional derivative of the Riemann curvature tensor $R$, seen as a symmetric double form has the form:

$$
R^{\prime} h=\text { Exact double form }+ \text { a linear term in } \mathrm{R},
$$

precisely,

$$
R^{\prime} h=-\frac{1}{4}(D \tilde{D}+\tilde{D} D)(h)+\frac{1}{4} F_{h}(R) .
$$


Next, we derive the directional derivative of the Gauss-Bonnet curvature $h_{2 k}$ at $g$ :

$$
h_{2 k}^{\prime} h=-\frac{1}{2}\left\langle\frac{c^{2 k-1}}{(2 k-1) !} R^{k}, h\right\rangle-\frac{k}{4}(\delta \tilde{\delta}+\tilde{\delta} \delta)\left(*\left(\frac{g^{n-2 k}}{(n-2 k) !} R^{k-1} h\right)\right),
$$

where $(\delta \tilde{\delta}+\tilde{\delta} \delta)$ is the formal adjoint of the Hessian type operator $(D \tilde{D}+\tilde{D} D)$.

Finally, using Stoke's theorem we conclude that:

$$
\begin{aligned}
H_{2 k}^{\prime} \cdot h & =\int_{M}\left(h_{2 k}^{\prime} \cdot h+\frac{h_{2 k}}{2} \operatorname{tr}_{g} h\right) \mu_{g}=-\frac{1}{2}\left\langle\frac{c^{2 k-1}}{(2 k-1) !} R^{k}, h\right\rangle+\frac{h_{2 k}}{2}\langle g, h\rangle \\
& =\frac{1}{2}\left\langle h_{2 k} g-\frac{c^{2 k-1}}{(2 k-1) !} R^{k}, h\right\rangle=\frac{1}{2}\left\langle T_{2 k}, h\right\rangle .
\end{aligned}
$$

\section{Applications}

\subsection{A generalized Yamabe problem [19]}

It results from the previous theorem (see [19]) that for a compact Riemannian $n$-manifold $(M, g)$ with $n>2 k$, the Gauss-Bonnet curvature $h_{2 k}$ is constant if and only if the metric $g$ is a critical point of the functional $H_{2 k}$ when restricted to the set $\operatorname{Conf}_{0}(g)$ of metrics pointwise conformal to $g$ and having the same total volume.

The previous result makes the following Yamabe-type problem plausible: In each conformal class of a fixed Riemannian metric on a smooth compact manifold with dimension $n>2 k$ there exists a metric with $h_{2 k}$ constant.

The previous problem is closely related to the recent $\sigma_{k}$-Yamabe problem of Viaclovsky involving the symmetric functions of the Schouten tensor, see [37, 34] and the references therein.

\subsection{Generalized Einstein manifolds [30, 19, 22]}

Einstein metrics are the critical metrics of the total scalar curvature functional once restricted to metrics of unit volume. Equivalently, a metric $g$ is Einstein if its Ricci tensor $c R$ is proportional to $g: c R=\lambda g$.

In a similar way, the critical metrics of the total Gauss-Bonnet curvature functional $H_{2 k}$ once restricted to metrics with unit volume shall be called $(2 k)$-Einstein metrics. The 2-Einstein metrics are nothing but the usual Einstein metrics.

These critical metrics were studied first by Patterson [30] who proved that a locally irreducible symmetric Riemannian metric is $(2 k)$-Einstein for any positive integer $k$, and that harmonic Riemannian metrics are 2 and 4-Einstein.

The previous $(2 k)$-Einstein metrics are characterized by the condition that the contraction of order $(2 k-1)$ of Thorpe's tensor $R^{k}$ is proportional to the metric, that is

$$
c^{2 k-1} R^{k}=\lambda g .
$$

More generally, for $0<p<2 q<n$, we shall say that a Riemannian $n$-manifold is $(p, q)$ Einstein [22] if the $p$-th contraction of Thorpe's tensor $R^{q}$ is proportional to the metric $g^{2 k-p}$, that is

$$
c^{p} R^{q}=\lambda g^{2 q-p} .
$$

We recover the usual Einstein manifolds for $p=q=1$ and the previous (2q)-Einstein condition for $p=2 q-1$. The $(p, q)$-Einstein metrics are all critical metrics for the total Gauss-Bonnet curvature functional $H_{2 q}$. 
For all $p \geq 1,(p, q)$-Einstein implies $(p+1, q)$-Einstein. In particular, the metrics with constant $q$-sectional curvature (that is the sectional curvature of $R^{q}$ is constant) are $(p, q)$ Einstein for all $p$.

On the other hand, the $(p, q)$-Einstein condition neither implies nor is implied by the $(p, q+1)$ condition as shown by the following examples.

Let $M$ be a 3-dimensional non-Einstein Riemannian manifold and $T^{k}$ be the $k$-dimensional flat torus, $k \geq 1$, then the Riemann curvature tensor $R$ of the Riemannian product $N=M \times T^{k}$ satisfies $R^{q}=0$ for $q \geq 2$. In particular $N$ is $(p, q)$-Einstein for all $p \geq 0$ and $q \geq 2$ but it is not $(1,1)$-Einstein.

On the other hand, let $M$ be a 4-dimensional Ricci-flat but not flat manifold (for example a $K_{3}$ surface endowed with the Calabi-Yau metric), then the Riemannian product $N=M \times T^{k}$ is $(1,1)$-Einstein but not $(q, 2)$-Einstein for any $q$ with $0 \leq q \leq 3$.

The $(2 q)$-Einstein condition, or equivalently the $(2 q-1, q)$-Einstein condition, seems to be so weak to imply any topological restrictions on the manifold. However, for lower values of $p$ we have the following obstruction result:

Theorem 2 ([22]). Let $k \geq 1$ and $(M, g)$ be a $(1, k)$-Einstein manifold (i.e. $c R^{q}=\lambda g^{2 q-1}$ ) of dimension $n \geq 4 k$. Then the Gauss-Bonnet curvature $h_{4 k}$ of $(M, g)$ is nonnegative. Furthermore, $h_{4 k} \equiv 0$ if and only if $(M, g)$ is $k$-flat.

In particular, a compact $(1, k)$-Einstein manifold of dimension $n=4 k$ has its Euler-Poincaré characteristic nonnegative. Furthermore, it is zero if and only if the metric is $k$-flat.

The previous theorem generalizes a similar result of Berger about usual four dimensional Einstein manifolds.

\section{$4.3 \quad(2 k)$-minimal submanifolds [23]}

Let $(\tilde{M}, \tilde{g})$ be an $(n+p)$-dimensional Riemannian manifold, and let $M$ be an $n$-dimensional submanifold of $\tilde{M}$.

We shall characterize those submanifolds (endowed with the induced metric) that are critical points of the total Gauss-Bonnet curvature function.

Let $F$ be a local variation of $M$, that is a smooth map

$$
F: M \times(-\epsilon, \epsilon) \rightarrow \tilde{M}
$$

such that $F(x, 0)=x$ for all $x \in M$ and with compact support $\operatorname{supp} F$.

The implicit function theorem implies that there exists $\epsilon>0$ such that for all $t$ with $|t|<\epsilon$, the map $\phi_{t}=F(\cdot, t): M \rightarrow \tilde{M}$ is a diffeomorphism onto a submanifold $M_{t}$ of $\tilde{M}$.

Let $g_{t}=\phi_{t}^{*}(\tilde{g})$. Note that $g_{1}=g$.

Theorem 3 ([23]). Let $\xi=\left.\frac{d}{d t}\right|_{t=0} \phi_{t}$ denotes the variation vector field relative to a local variation $F$ of $M$ with compact support as above.

1. If $H_{2 k}(t)=\int_{M} h_{2 k}\left(g_{t}\right) \mu_{g_{t}}$ denotes the total (2k)-th Gauss-Bonnet curvature of $\phi_{t}(M)$, then

$$
H_{2 k}^{\prime}(0)=\int_{M} h_{2 k+1}\left(\xi^{\perp}\right) \mu_{g}
$$

where $h_{2 k+1}$ is the $(2 k+1)$-th Gauss-Bonnet curvature of $M$ defined by (2).

2. The submanifold $M$ is a critical point for the total (2k)-th Gauss-Bonnet curvature function for all local variations of $M$ if and only if the $(2 k+1)$-Gauss-Bonnet curvature $h_{2 k+1}(N)$ of $M$ vanishes for all normal directions $N$. 
With reference to the previous variational formula and by analogy to the case of usual minimal submanifolds we set the following definition:

Definition 2. For $0 \leq 2 k \leq n$, an $n$-submanifold $M$ of a $\operatorname{Riemannian~manifold~}(\tilde{M}, \tilde{g})$ is said to be $(2 k)$-minimal if $h_{2 k+1} \equiv 0$.

Note that since $h_{2 k+1}(N)=\left\langle T_{2 k}, B_{N}\right\rangle$, a submanifold is $(2 k)$-minimal if and only if $T_{2 k}$ is orthogonal to $B_{N}$ for all normal directions $N$. Note the analogy with usual minimal submanifolds $\left(T_{0}=g\right)$.

We list below some examples:

1. A flat submanifold is always $(2 k)$-minimal for all $k>0$. In fact $R \equiv 0 \Rightarrow h_{2 k+1} \equiv 0$. This shows that $(2 k)$-minimal does not imply the usual minimality condition.

2. A totally geodesic submanifold is always $(2 k)$-minimal for all $k \geq 0$. In fact $B \equiv 0 \Rightarrow$ $h_{2 k+1} \equiv 0$.

3. If $M$ is a hypersurface of the Euclidean space then $(2 k)$-minimality coincides with Reilly's (2k)-minimality, [31]. On the other hand, if $M$ is a hypersurface of a space form $(\tilde{M}, \tilde{g})$ of constant $\lambda$ then $M$ is $(2 k)$-minimal if and only if

$$
\sum_{i=0}^{k} \frac{(2 k-2 i+1) !(n-2 k-1+2 i) ! \lambda^{i}}{i !(k-i) !} s_{2 k-2 i+1}=0 .
$$

In particular, $M$ is 2 -minimal if and only if $6 s_{3}+(n-1)(n-2) s_{1} \lambda=0$. Notice the difference with Reilly's $r$-minimality.

4. A complex submanifold $M$ of a Kahlerian manifold $(\tilde{M}, \tilde{g})$ is $(2 k)$-minimal for any $k$.

Let now $f$ be a smooth function on $(M, g)$. We define the $\ell_{2 k}$-Laplacian [23] operator of $(M, g)$ as

$$
\ell_{2 k}(f)=-\left\langle T_{2 k}, \operatorname{Hess}(f)\right\rangle
$$

where $T_{2 k}$ denotes the $(2 k)$-th Einstein-Lovelock tensor $(4)$ of $(M, g)$ and $0 \leq 2 k<n$, Hess $(f)$ is the Hessian of $f$.

For $k=0$ we have $T_{0}=g$ and then $\ell_{0}=\Delta$ is the usual Laplacian.

For a compact manifold, the generalized Laplacian $\ell_{2 k}$ satisfies the following interesting properties:

For each $k \geq 0, \ell_{2 k}(f)$ is a divergence hence $\int_{M} \ell_{2 k}(f) d v \equiv 0$. Furthermore, the operator $\ell_{2 k}$ is self adjoint with respect to the integral scalar product.

If for some $k$ with $0 \leq 2 k<n$, the Einstein-Lovelock tensor $T_{2 k}$ is positive definite (or negative definite), then the operator $\ell_{2 k}$ is elliptic and positive definite (resp. negative definite).

We shall say that the function $f$ is $\ell_{2 k}$-harmonic if $\ell_{2 k}(f)=0$. In [23] we proved the following maximum principle:

Theorem 4 ([23]). Let $(M, g)$ be a compact manifold of positive definite (or negative definite) Einstein-Lovelock tensor $T_{2 k}$ then every smooth and $\ell_{2 k}$-harmonic function on $M$ is constant.

As a consequence of the previous result we proved the following about $(2 k)$-minimal submanifolds of the Euclidean space:

Theorem 5. A submanifold $M$ of the Euclidean space is $(2 k)$-minimal if and only if the coordinate functions restricted to $M$ are $\ell_{2 k}$-harmonic functions on $M$. 
Corollary 1. Let $0 \leq 2 k<n$ and let $(M, g)$ be a compact Riemannian $n$-manifold with positive definite (or negative definite) Einstein-Lovelock tensor $T_{2 k}$. Then there is no non trivial isometric (2k)-minimal immersion of $M$ into the Euclidean space.

Note that the condition of positive (or negative) definiteness of $T_{2 k}$ in the previous corollary is necessary, as the flat torus admits (non trivial) (2k)-minimal isometric immersions into the Euclidean space.

\section{Acknowledgments}

The author would like to thank the referees for useful comments and especially for indicating me the related work of Patterson [30].

\section{References}

[1] Adler R.J., On excursion sets, tube formulas and maxima of random fields, Ann. Appl. Probab. 10 (2000), $1-74$.

[2] Albin P., Renormalizing curvature integrals on Poincaré-Einstein manifolds, math.DG/0504161.

[3] Berger M., Quelques formules de variation pour une structure riemannienne, Ann. Sci. Ecole Norm. Sup. (4) 3 (1970), 285-294.

[4] Bernig A., Variations of curvatures of subanalytic spaces and Schläfli-type formulas, Ann. Global Anal. Geom. 24 (2003), 67-93.

[5] Bernig A., On some aspects of curvature, available at http://homeweb1.unifr.ch/BernigA/pub/.

[6] Besse A.L., Einstein manifolds, Springer-Verlag, Berlin, 1987.

[7] Bourguignon J.P., Les variétés de dimension 4 à signature non nulle dont la courbure est harmonique sont d'Einstein, Invent. Math. 63 (1981), 263-286.

[8] Cai R.G., Ohta N., Black holes in pure lovelock gravities, Phys. Rev. D 74 (2006), 064001, 8 pages, hep-th/0604088.

[9] Cheeger J., Müller W., Schräder R., On the curvature of piecewise flat spaces, Comm. Math. Phys. 92 (1984), 405-454.

[10] Deruelle N., Madore J., On the quasi-linearity of the Einstein-Gauss-Bonnet gravity field equations, gr-qc/0305004.

[11] Fenchel W., On total curvatures of Riemannian manifolds. I, J. Lond. Math. Soc. 15 (1940), 15-22.

[12] Gray A., Some relations between curvature and characteristic classes, Math. Ann. 184 (1970), $257-267$.

[13] Gray A., Tubes, Progress in Mathematics, Vol. 221, Birkhäuser Verlag, Basel, 2004.

[14] Ishihara T., Kinematic formulas for Weyl's curvature invariants on submanifolds in complex projective space, Proc. Amer. Math. Soc. 97 (1986), 483-487.

[15] Kowalski O., On the Gauss-Kronecker curvature tensors, Math. Ann. 203 (1973), 335-343.

[16] Kulkarni R.S., On Bianchi identities, Math. Ann. 199 (1972), 175-204.

[17] Labbi M.L., Double forms, curvature structures and the $(p, q)$-curvatures, Trans. Amer. Math. Soc. 357 (2005), 3971-3992, math.DG/0404081.

[18] Labbi M.L., On compact manifolds with positive second Gauss-Bonnet curvature, Pacific J. Math. 227 (2006), 295-310.

[19] Labbi M.L., Variational properties of the Gauss-Bonnet curvatures, in Calculus of Variations and Partial Differential Equations, to appear, math.DG/0406548.

[20] Labbi M.L., Riemannian curvature: variations on different notions of positivity, math.DG/0611371.

[21] Labbi M.L., On Weitzenböck curvature operators, math.DG/0607521.

[22] Labbi M.L., Remarks on generalized Einstein manifolds, math.DG/0703028.

[23] Labbi M.L., On (2k)-minimal submanifolds, arXiv:0706.3092. 
[24] Lafontaine J., Mesures de courbure des variétés lisses et des polyèdres [d'après Cheeger, Müller et Schräder], Séminaire Bourbaki, 38ème année, 1985-86, no. 664, 1986.

[25] Lovelock D., The Einstein tensor and its generalizations, J. Math. Phys. 12 (1971), 498-501.

[26] Madore J., Cosmological applications of the Lanczos Lagrangian, Classical Quantum Gravity 3 (1986), 361-371.

[27] Muto Y., Critical Riemannian metrics, Tensor 29 (1975), 125-133.

[28] Nasu T., On conformal invariants of higher order, Hiroshima Math. J. 5 (1975), 43-60.

[29] Nomizu K., On the decomposition of generalized curvature tensor fields. Codazzi, Ricci, Bianchi and Weyl revisited, in Differential Geometry (in Honor of Kentaro Yano), Kinokuniya, Tokyo, 1972, 335-345.

[30] Patterson E.M., A class of critical Riemannian metrics, J. London Math. Soc. 2 (1981), 349-358.

[31] Reilly R.C., Variational properties of functions of the mean curvatures for hypersurfaces in space forms, J. Differential Geom. 8 (1973), 465-477.

[32] Reilly R.C., Variational properties of mean curvatures, in J. Proc. Summer Sem. Canad. Math. Congress, 1971, 102-114.

[33] Senovilla J.M.M., Super-energy tensors, Classical Quantum Gravity 17 (2000), 2799-2842, gr-qc/9906087.

[34] Sheng W., Trudinger N.S., Wang X.-J., The Yamabe problem for higher order curvatures, J. Differential Geom., to appear.

[35] Stehney A., Courbure d'ordre $p$ et les classes de Pontrjagin, J. Differential Geom. 8 (1973), 125-134.

[36] Thorpe J.A., Some remarks on the Gauss-Bonnet integral, J. Math. Mech. 18 (1969), 779-786.

[37] Viaclovsky J., Conformal geometry and fully nonlinear equations, in World Scientific Memorial Volume for S.S. Chern, to appear, math.DG/0609158.

[38] Weyl H., On the volume of tubes, Amer. J. Math. 61 (1939), 461-472. 